\title{
Establishment of PEEK-Associated Drug Delivery Systems-Limits and Perspectives
}

\author{
Marius Behnecke, Svea Petersen* \\ Laboratory for Chemistry and Surface Modification, Hochschule Osnabrück University of Applied Sciences, Osnabrück, Germany \\ Email: *s.petersen@hs-osnabrueck.de
}

How to cite this paper: Behnecke, M. and Petersen, S. (2020) Establishment of PEEKAssociated Drug Delivery Systems-Limits and Perspectives. Journal of Materials Science and Chemical Engineering, 8, 32-41.

https://doi.org/10.4236/msce.2020.810004

Received: August 31, 2020

Accepted: October 26, 2020

Published: October 29, 2020

Copyright $\odot 2020$ by author(s) and Scientific Research Publishing Inc. This work is licensed under the Creative Commons Attribution International License (CC BY 4.0).

http://creativecommons.org/licenses/by/4.0/

\begin{abstract}
In order to enhance promising properties of polyethertherketone (PEEK) for biomedical application, we evaluate two different strategies for the provision of PEEK with a drug delivery system at the example of the model drug chlorhexidine diacetate. On one hand we discuss the deposition of a biodegradable polymeric drug-release coating, based on well-established poly(L-lactide) (PLLA), on PEEK and on the other hand the modification of PEEK via sulfonation and its subsequent drug loading. Results are compared to pure PLLA-associated drug delivery systems and limits and perspectives are shown.
\end{abstract}

\section{Keywords}

Polyethertherketone, Drug Chlorhexidine

\section{Introduction}

Polyetheretherketone (PEEK) has been originally created in 1978 by a group of British researchers [1]. Already beginning in the early 1980s, PEEK has been widely used in modern applications, such as aviation, ships and turbine edges [2]. By the late 1990s, PEEK entered the medical sector and became a promising polymer alternative to metal implant components, especially in orthopedic and traumatic applications but also in dentistry. In addition to aesthetics, the benefit of using PEEK instead of metal components is the amazing biomechanical property. While traditionally used dental and orthopedic implant materials (such as titanium) have a relatively high Young's modulus of $116 \mathrm{GPa}$ compared to bone [3], the Young's modulus of PEEK is about 3.6 GPa, which can be enhanced to $18 \mathrm{GPa}$ by means of fibre reinforcement [4]. As cortical bone evidences a comparable elastic modulus [5] [6], stress shielding is supposed to be lower for PEEK compared to titanium. Moreover, PEEK has been proven to be 
biocompatible. No toxic or mutagenic effects or inflammation were reported [3] [7]. However, PEEK is biologically inert [8] [9], which limits its potential applications. One possibility to overcome this obstacle could be the provision of PEEK with a drug delivery system. While certain drug delivery systems are already used in orthopaedics and dentistry, as for example the Septocoll E-fleece [10], characterized by a metabolizing collagen matrix containing gentamicin as a preventive local antibiotic protection, and the PerioChip persisting of a gelatine matrix with the antibacterial chlorhexidine gluconate, PEEK-associated drug delivery systems have not been described so far. The main challenge is probably that most bioactive components are not able to stand to processing conditions of PEEK, being either high melting temperature $>350^{\circ} \mathrm{C}$ or strongly acidic solvents as hydrofluoric acid. Within this study we aim at the evaluation of different strategies for the provision of PEEK with a delivery system of the model drug chlorhexidine diacetate. On one hand we discuss the deposition of a biodegradable polymeric drug-release coating, based on well-established poly(L-lactide) (PLLA), on PEEK and on the other hand the modification of PEEK via sulfonation and its subsequent drug loading. Results are compared to pure PLLA-associated drug delivery systems and limits and perspectives are shown.

\section{Experimental}

\subsection{Materials}

PEEK foils (thickness: $1 \mathrm{~mm}$ ) and pellets (VESTAKEEP 4000G) were kindly provided by Evonik Industries AG. PLLA (RESOMER ${ }^{\bullet}$ L $10 \mathrm{~S}$ ) was also provided by Evonik Industries AG. In this study chlorhexidine diacetate (CHX) was used as a model drug which was purchased from Caesar \& Lorentz GmbH (Hilden, Germany). All solvents and other chemicals used were purchased from Sigma Aldrich.

\subsection{Sample Preparation}

\subsubsection{Preparation of Pure PLLA Drug Delivery Films by Solvent Evaporation Method}

PLLA films is produced by the solvent casting process. The model release systems from PLLA are designed to have an active ingredient content of $5 \%$ and a film thickness of approximately $100 \mu \mathrm{m}$. For this purpose, in a $25 \mathrm{~mL}$ volumetric flask $950 \mathrm{mg}$ PLLA was dissolved in $24 \mathrm{~mL} \mathrm{CHCl}_{3}$ and the flask was filled up to $25 \mathrm{~mL}$ with a $50 \mathrm{mg} / \mathrm{mL} \mathrm{CHX} /$ methanol stock solution. The solution was homogenized on an orbital shaker and then transferred to a $9 \mathrm{~cm}$ petri dish. The solvent was evaporated at room temperature and covered with a perforated parafilm to slow down the evaporation rate and thereby receive homogeneous films. After three days under the laboratory hood, the resulting films were finally dried at $100 \mathrm{mbar}$ and $40^{\circ} \mathrm{C}$ in a vacuum drying oven.

\subsubsection{Modification of PEEK by Means of EDA}

With the purpose of improving the wettability of provided PEEK foils for sub- 
sequent deposition of a PLLA coating, the PEEK surface was modified via an amination procedures using ethylenediamine (EDA). Therefore, PEEK film specimen were cut, cleaned in an ultrasonic bath with isopropanol and then dried at room temperature. Following Becker et al., the modification was performed in a single step by treating the foils for 3 hours with EDA in a reflux apparatus at $120^{\circ} \mathrm{C}$ [11]. After the amination procedures PEEK films were dried in vacuum at $60^{\circ} \mathrm{C}$ overnight and stored in a desiccator until further use.

\subsubsection{Deposition of PLLA-Coating via Dip-Coating}

Subsequently, both aminated and untreated PEEK surfaces were coated with PLLA via a dip-coating procedure. Therefore, PEEK samples were dipped 5 times into a polymer solution (0.5\% PLLA in chloroform with $0.025 \%$ CHX, previously dissolved in methanol). Between those dipping cycles the drying time was ten minutes.

\subsubsection{Preparation of Sulfonated PEEK Specimen}

The sulfonation of PEEK was performed by concentrated sulfuric acid. Therefore $2 \mathrm{~g}$ granulated PEEK was immersed in $50 \mathrm{~mL}$ sulfuric acid (98\%, Sigma-aldrich) and stored on a magnetic stirrer for $48 \mathrm{~h}$ and $72 \mathrm{~h}$. After the sulfonation process the dissolved now sulfonated PEEK (sPEEK) was then precipitated in $2^{\circ} \mathrm{C}$ cold, demineralized water. The precipitation was performed by transferring the sPEEK-solution in drops via a drip funnel in the cooled water. After filtration the material was washed with demineralized water several times until the solution reached $\mathrm{pH}>5$. The material was then dried in a vacuum oven at 100 mbar for 12 hours. Just like the PLLA films, sPEEK films were made via solvent casting method. To produce films with the desired CHX content and film thickness $0.076 \mathrm{~g}$ of produced sPEEK was resolved in $1.9 \mathrm{~mL}$ dimethylformamide (DMF). After the polymer was completely dissolved $0.1 \mathrm{~mL}$ of a 40 $\mathrm{mg} / \mathrm{L} \mathrm{CHX} / \mathrm{DMF}$-solution was added and the solution was homogenized. For film production the solution was casted in a $30 \mathrm{~mm}$ petri dish and the solvent was evaporated at $35^{\circ} \mathrm{C}$ over three days. The direct loading of sPEEK-droplets was performed by immersion of the droplets in a $40 \mathrm{mg} / \mathrm{L}$ aqueous $\mathrm{CHX}$-solution over $12 \mathrm{~h}$, followed by a short washing with demineralized water and subsequent drying in a vacuum oven at $100 \mathrm{mbar}$ and $40^{\circ} \mathrm{C}$.

\subsection{Characterization Methods}

\subsubsection{Electronmicroscopy}

A topographical analysis of the different types of CHX-drug delivery systems was performed via scanning electron microscopy (SEM). To avoid damaging of the polymeric surfaces the samples were sputtered with platinum-iridium alloy and low acceleration voltages between 1 and $3 \mathrm{kV}$ were used. The analysis was performed on a dual-beam SEM "AURIGA"-system manufactured by Carl Zeiss AG (Oberkochen, Germany), using magnifications ranging from 1.000 to 5.000 times. 


\subsubsection{Water Contact Angle Measurements}

The contact angles of the differently modified PEEK samples were measured using a OCA 20 goniometer by dataphysics (Filderstadt, Germany). In addition, the manufacturer's SCA20 software was used to perform drop contour analysis, define a baseline, and calculate the resulting angles. The only test liquid used was water to evaluate the hydrophilicity of the sample surfaces. To determine the contact angle, the sessile drop method was used. For this purpose, a droplet of 1 $\mu \mathrm{L}$ was dosed onto the surface and after 10 seconds the contact angle at the three-phase point was measured.

\subsubsection{FTIR-Spectroscopy}

To ensure the successful sulfonation of PEEK infrared spectroscopy was performed on each sample. Therefore, the ATR-method was used on an FTIR-system "AVATAR 360" by Thermo Fisher Scientific Inc. (Waltham, USA) with the device designation. For spectra acquisition and analysis, the manufacturers "OMNIC 8.3" software was used. For each measurement 32 single scans are performed, analyzing the wave number range from $4000 \mathrm{~cm}^{-1}$ to $600 \mathrm{~cm}^{-1}$.

\subsubsection{Degree of Sulfonation}

The percentage of substituted carbonyl groups is referred to the materials "sulfonation degree" (DS). To determine this parameter different methods can be used, in this case it was analyzed by titration. For this purpose, a cation exchange on the prepared sPEEK was performed. This was done by immersing $0.2 \mathrm{~g}$ sPEEK in $50 \mathrm{~mL} 2 \mathrm{M} \mathrm{NaCl}$-solution for 24 hours. After filtration the resulting solution can be titrated to the equivalence point against a $0.05 \mathrm{M}$ sodium hydroxide. Based on the volume needed for the neutralization of the solution according to Huang et al. the DS can be calculated using the following equation [12]:

$$
\mathrm{DS}=\frac{n\left(\mathrm{SO}_{3}^{-}\right) \cdot M(\mathrm{PEEK})}{m(\mathrm{PEEK})-n\left(\mathrm{SO}_{3}^{-}\right) \cdot M\left(\mathrm{SO}_{3}^{-}\right)}
$$

\subsubsection{In Vitro Drug Release Studies}

On all samples an "in-vitro" drug release study was performed to quantify the amount of released CHX over time. For this purpose, $12 \mathrm{~mm}$ circular plates were carefully die-cut from the samples when necessary. These plates were immersed in phosphate-buffered salt solution according to Sörensen as release medium and tempered to $37^{\circ} \mathrm{C}$. After defined time intervals, which increase during the examination, the solution is drawn off and renewed. The solution is then analyzed by HPLC to determinate the amount of CHX. By cumulating the determined masses of $\mathrm{CHX}$ the systems drug release over time can be characterized.

\subsubsection{HPLC Parameters}

To quantify the released amount of CHX the release medium is analyzed by HPLC. The column used is a C18 column called "Poroshell 120 EC-C18" (Agilent Technologies, Inc., Santa Clara, USA) with a pore size of 2.7 micrometers, a 
column diameter of $3 \mathrm{~mm}$ and a length of $50 \mathrm{~mm}$. The mobile phase used was methanol and Mcllvaine citric acid-phosphate buffer ( $\mathrm{pH} 2.3$ ) at a ratio of 60/40 at a flow rate of $0.3 \mathrm{~mL} / \mathrm{min}$. Per measurement $2 \mu \mathrm{L}$ sample were injected, the temperature of the column oven during the measurements was $20^{\circ} \mathrm{C}$. To calibrate the method, a dilution series is prepared from a CHX stock solution with a concentration of $1 \mathrm{mg} / \mathrm{mL}$. For this purpose, $10 \mathrm{mg}$ CHX were dissolved in 10 $\mathrm{mL}$ mobile phase and homogenized in an ultrasonic bath for 5 minutes. Further dilution is also carried out in mobile phase and 5 minutes in the ultrasonic bath. The concentration range of $5-95 \mu \mathrm{g} / \mathrm{mL}$ is calibrated with a total of 7 calibration points at $15 \mu \mathrm{g} / \mathrm{mL}$ intervals.

\section{Results and Discussion}

The provision of PEEK with a drug delivery function is a promising approach to enhance biocompatibility of PEEK-based medical applications. Within this study, we discuss two approaches for preparation. The first approach is the deposition of a polymer coating with drug incorporation on the surface of PEEK and the second approach is the sulfonation of PEEK in order to modify its solubility properties and thereby allow for drug incorporation within solution and subsequent film preparation via solvent casting.

\subsection{Preparation of a Surface-Coated PEEK-Associated Drug Delivery System}

The successful and stable deposition of a polymeric coating on PEEK affords previous surface modification as we have previously published [13]. In here, we applied a comparable method via amination of the PEEK surface with ethylendiamine and subsequent PLLA deposition via dip coating. Results evidenced better adhesive strength of the PLLA coating to aminated PEEK compared to unmodified PEEK. While most parts of the coating were pulled off from unmodified PEEK surfaces during a grid scratch test, the coating on aminated surfaces hardly show any defects after elimination of the adhesive tape. The provided coating was adjusted by means of dipping intervals to approximately $5 \mu \mathrm{m}$.

\subsection{Preparation of Bulk sPEEK-Associated Drug Delivery System}

The sPEEK was produced using concentrated sulfuric acid with subsequent quenching in an ice bath. The precipitation as droplets using a dropping funnel made it possible to obtain a homogeneous material without the formation of fine particles, which were lost during cleaning. Thus, we reached a recovery of $\geq 90 \%$ of used PEEK raw pellets. The characterization using FTIR (Figure 1) was able to confirm the successful sulfonation of the polymer by bands characteristic of the sulfonic acid group $\left(1080 \mathrm{~cm}^{-1}\right.$, symmetric $\mathrm{O}=\mathrm{S}=\mathrm{O}$ vibration). That these peaks were only caused by a residual amount of $\mathrm{H}_{2} \mathrm{SO}_{4}$ can be excluded via a peak that indicates a new substituent on an aromatic ring $\left(1480 \mathrm{~cm}^{-1}\right)$. In addition, a bond between carbon and sulfur could be proven via a band at $630 \mathrm{~cm}^{-1}$ for C-S vibration. The back titration of the sulfonic acid groups also showed the 


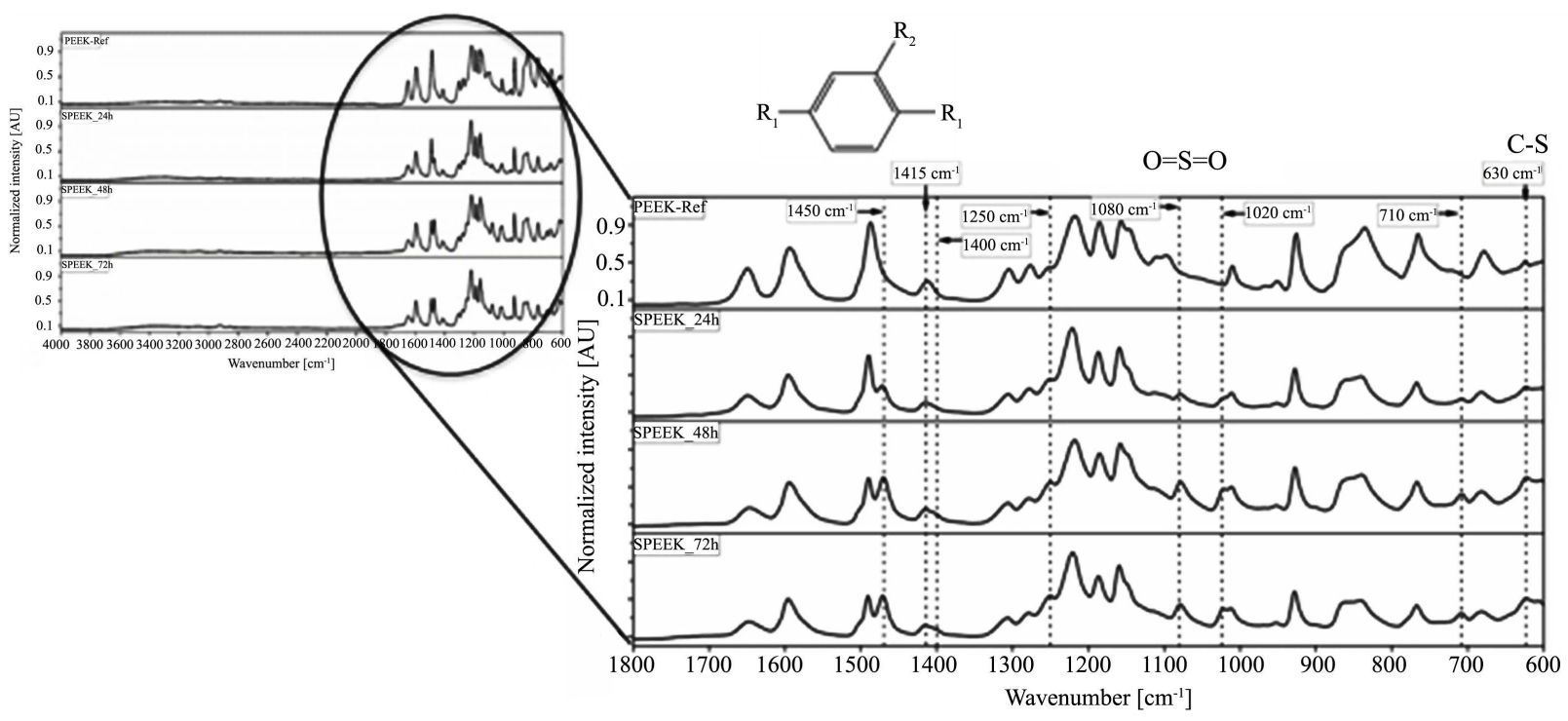

Figure 1. FTIR-spectra of PEEK after different times of sulfonation (PEEK-Ref = unmodified PEEK, PEEK_24 h, PEEK_48 h, PEEK_72 $\mathrm{h}=$ PEEK after $\mathrm{x}$ hours of sulfonation).

expected results with increasing degree of sulfonation with increasing sulfonation time (Table 1) being in accordance to literature reports [12] [14]. The solubility of sPEEK in different solvents is a decisive factor for the production of a drug delivery system without damaging the active ingredient, but it can also be seen as a further indication of the successful modification. For all sulfonation times, we could observe solubility in DMF, dimethylsufoxide (DMSO) and N-Methyl-2-pyrrolidone (NMP), also corresponding to representations in literature for the solubility of sPEEK [15]. The solubility in DMSO is promising, as this solvent is already used for the preparation of medical application. However, due to the very low vapour pressure of DMSO, DMF with a higher vapour pressure was used for further processing of sPEEK films by solvent casting. Moreover, solubility and stability of CHX could be proven in DMF, allowing for the preparation of $\mathrm{CHX}$-containing sPEEK films via solvent casting.

\subsection{Morphology of PEEK-Associated Drug Delivery Systems}

Examination of reference films of PLLA-coatings and sPEEK-films without drug incorporation by means of scanning electron miscroscopy reveals a different appearance. While sPEEK surfaces are rather smooth except manufacturing-related scratches (Figure 2(d)), PLLA-coatings are rougher with spherical domains homogenously spread over the surface (Figure $2(\mathrm{c})$ ), probably caused via solvent evaporation during the dip-coating process. The integration of CHX leads in sPEEK films (Figure 2(b)) to the appearance of surface-attached small domains and in PLLA-coatings to the multiplication of spherical domains (Figure 2(a)).

\subsection{Wettability of PEEK-Associated Drug Delivery Systems}

Looking at the results of the contact angle measurements (Figure 3), one gets the 


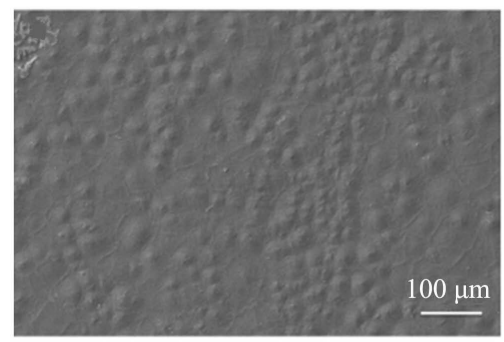

(a)

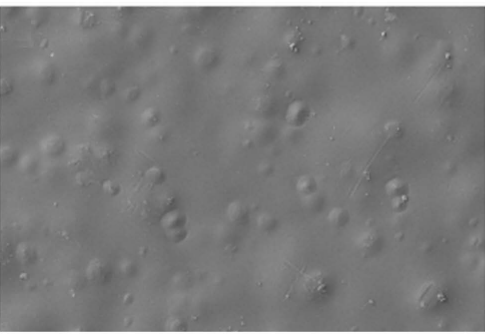

(b)

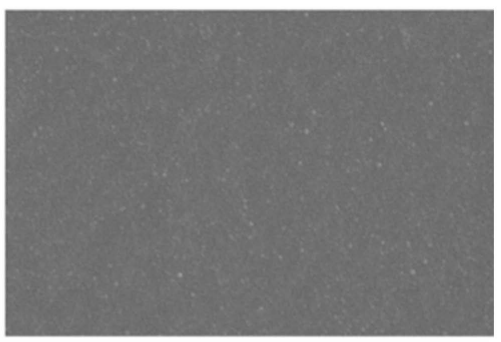

(c)

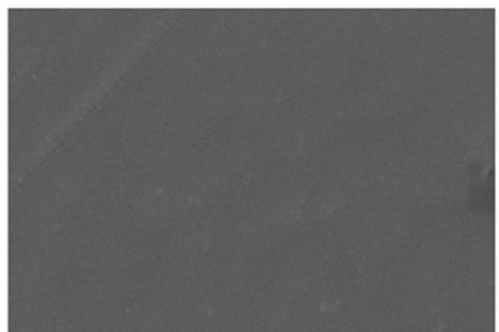

(d)

Figure 2. Scanning electron micrographs of PLLA ((a), (c)) and PEEK ((b), (d)) films with ((a), (b)) and without ((c), (d)) chlorhexidine diacetate integration.

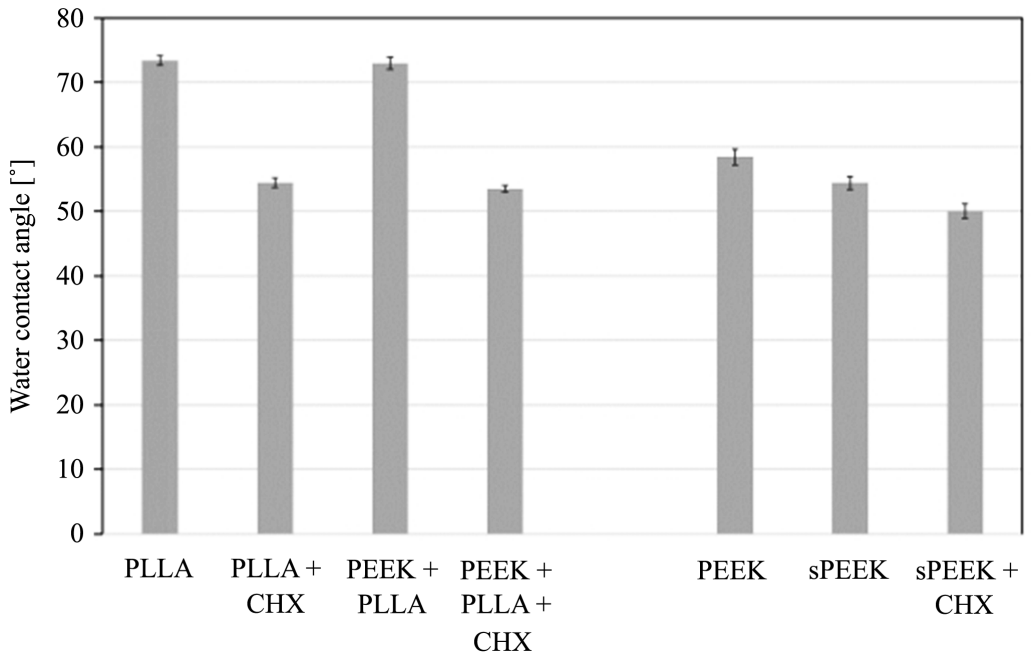

Figure 3. Water contact angle of different drug delivery systems. Bars evidence mean \pm standard deviation of $\mathrm{n}>9$.

Table 1. Degree of sulfonation of PEEK treated different hours with $\mathrm{H}_{2} \mathrm{SO}_{4}$ as determined via titration.

\begin{tabular}{cc}
\hline Time of sulfonation $[\mathrm{h}]$ & Degree of sulfonation [\%] \\
\hline 24 & 21.8 \\
48 & 48.6 \\
72 & 63.8 \\
\hline
\end{tabular}

impression that the CHX loading enhances hydrophilicity of the PLLA-coating as well as the sPEEK-films. As CHX is rather polar due to the presence of amines and chlorine, these data suggest the existence of CHX on the surface of poly- 
mers, being in accordance with the observation of increasing surface roughness by scanning electron micrographs.

\subsection{In Vitro Drug Release of PEEK-Associated Drug Delivery Systems}

The drug release profile from PLLA-coatings corresponds to the expected behavior of a diffusion-controlled drug delivery system. A first burst release is followed by a slow long-term release phase (Figure 4). The reduction of the amount of drug released for PLLA-coatings on PEEK foils in comparison to pure PLLA films can be probably dedicated to the halved surface contact to the surrounding media. Independent of the sulfonation time, no drug release could be detected neither for sPEEK films in the common release medium phosphate buffered saline nor in other solvents as methanol or pure double distilled water (results not shown). The authors can only speculate on reasons: most likely is a complex formation between the positive amine charge in chlorhexidine diacetate and the negatively charged sulfongroups in SPEEK, which hinders detachment of drug from the sPEEK matrix. Less possible reasons are the instability of $\mathrm{CHX}$ due to remaining sulfuric acid in the polymer matrix, which could however not be proven by means of FTIR, or the decomposition of CHX in DMF, which would be in contradiction to performed solvent stability assays by means of HPLC analysis. Besides the preparation of drug-loaded sPEEK-films we directly loaded sPEEK droplets as obtained after precipitation with CHX (Figure 5(b) and Figure 5(c)) via simple immersion. Here, drug release could be detected with nearly $10 \%$ of drug released after five hours (Figure 5(a)). Obviously, CHX did not interpenetrate within the droplets and could be easily released from the surfaces.

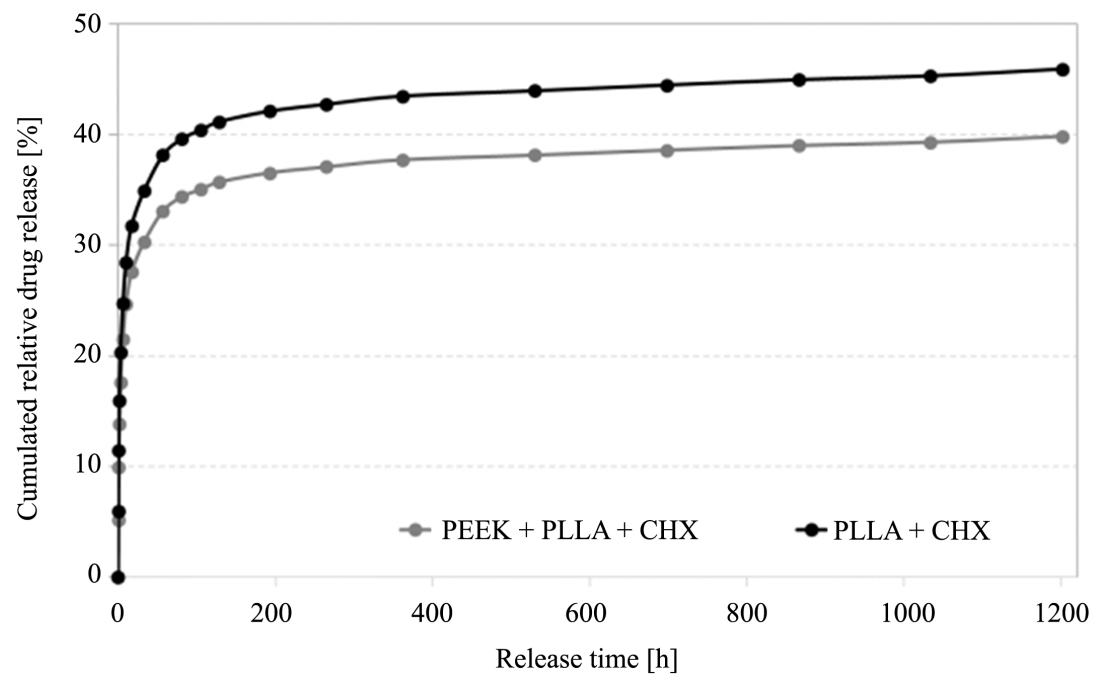

Figure 4. Cumulated relative drug release of PLLA-based and PEEK-associated PLLA-based CHX release systems. Results are shown as means of $\mathrm{n}>3$. In vitro drug release was performed in phosphate buffered saline, $\mathrm{pH}=7.2,37^{\circ} \mathrm{C}$ under continuous shaking. 


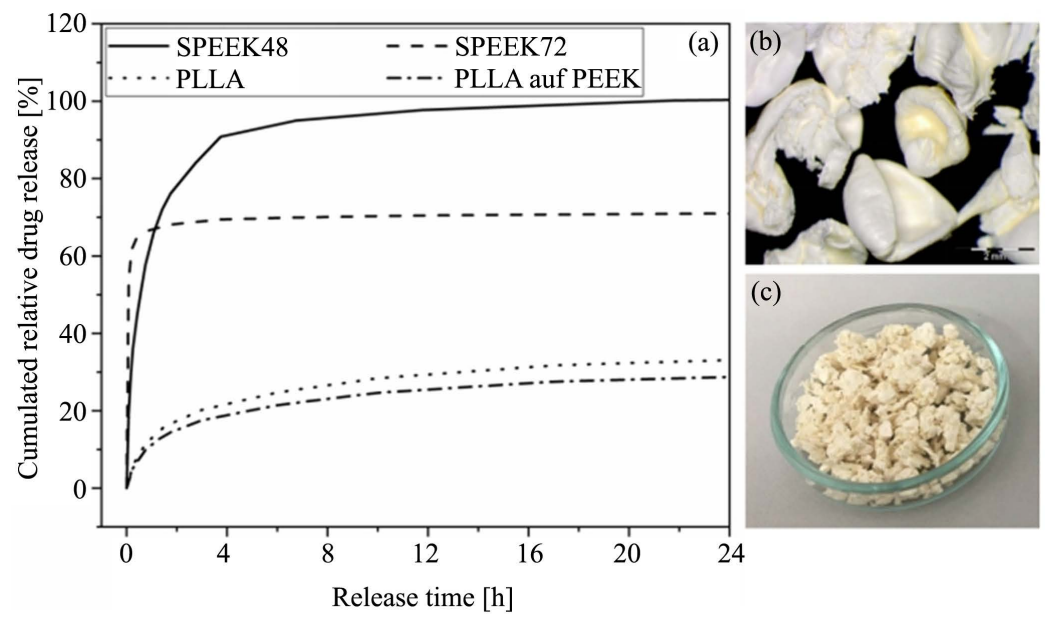

Figure 5. (a) Cumulated relative drug release of sPEEK-droplet-based CHX release systems. Results are shown as means of $\mathrm{n}>3$ in comparison to drug release profiles of PLLA and PEEK-associated PLLA-based CHX release systems. In vitro drug release was performed in each case phosphate buffered saline, $\mathrm{pH}=7.2,37^{\circ} \mathrm{C}$ under continuous shaking. (b) microscopic and (c) photograph of sPEEK-droplets after 48 hour of sulfonation.

\section{Conclusion}

Summarizing, the establishment of PEEK-associated drug delivery systems is as assumed challenging. In this study successful drug release could be observed from PEEK coated with a CHX-containing biodegradable PLLA-coating. However, the authors assume that the application range would be limited due to possible delamination of the coating during insertion of the medical application. Bulk modification could be therefore more promising. In here, we investigated the sulfonation of PEEK with subsequent drug loading by means of film preparation via solvent casting or by means of direct immersion of prepared sPEEK-droplets in drug solution. Unfortunately, we were not able to observe drug release from sPEEK films, probably due to stable binding of CHX within the sPEEK-matrix. sPEEK-droplets allowed for a very rapid release, which proves the principal possibility drug release from PEEK without surface coating.

\section{Acknowledgements}

The authors acknowledge the technical support of Veronika Terveen, Hannelore Schmidt, Felix Sander, Regina Pfefferle, Eva Jerman and Katharina Mey, who assisted during the characterization of the established drug release systems. Moreover, the provision of PEEK from Evonik Materials is appreciated.

\section{Conflicts of Interest}

The authors declare no conflicts of interest regarding the publication of this paper.

\section{References}

[1] Eschbach, L. (2000) Nonresorbable Polymers in Bone Surgery. Injury, 31, D22-D27. 
https://doi.org/10.1016/S0020-1383(00)80019-4

[2] Margolis (1985) Engineering Thermoplastics: Properties and Applications. CRC Press, Boca Raton.

[3] Wenz, L.M., Merritt, K., Brown, S.A., Moet, A. and Steffee, A.D. (1990) In Vitro Biocompatibility of Polyetheretherketone and Polysulfone Composites. Journal of Biomedical Materials Research, 24, 207-215. https://doi.org/10.1002/jbm.820240207

[4] Isidor, F. (2006) Influence of Forces on Peri-Implant Bone. Clinical Oral Implants Research, 17, 8-18. https://doi.org/10.1111/j.1600-0501.2006.01360.x

[5] Godara, A., Raabe, D. and Green, S. (2007) The Influence of Sterilization Processes on the Micromechanical Properties of Carbon Fiber-Reinforced PEEK Composites for Bone Implant Applications. Acta Biomaterialia, 3, 209-220. https://doi.org/10.1016/j.actbio.2006.11.005

[6] Williams, D. (2008) Polyetheretherketone for Long-Term Implantable Devices. Medical Device Technologies, 19, 10-11.

[7] Nieminen, T., Kallela, I., Wuolijoki, E., Kainulainen, H., Hiidenheimo, I. and Rantala, I. (2008) Amorphous and Crystalline Polyetheretherketone: Mechanical Properties and Tissue Reactions during a 3-Year Follow-Up. Journal of Biomedical Materials Research Part A, 84A, 377-383. https://doi.org/10.1002/jbm.a.31310

[8] Briem, D., Strametz, S., Schröder, K., Meenen, N.M., Lehmann, W., Linhart, W., Ohl, A. and Rueger, J.M. (2005) Response of Primary Fibroblasts and Osteoblasts to Plasma Treated Polyetheretherketone (PEEK) Surfaces. Journal of Materials Science: Materials in Medicine, 16, 671-677. https://doi.org/10.1007/s10856-005-2539-Z

[9] Wang, H., Xu, M., Zhang, W., Kwok, D.T.K., Jiang, J., Wu, Z. and Chu, P.K. (2010) Mechanical and Biological Characteristics of Diamond-Like Carbon Coated Poly Aryl-Ether-Ether-Ketone. Biomaterials, 31, 8181-8187.

https://doi.org/10.1016/j.biomaterials.2010.07.054

[10] Holzer, B., Grüssner, U., Brückner, B., Houf, M., Kiffner, E., Schildberg, F.W., Vogel, P., Rosen, H.R. and EMD Study Group (2003) Efficacy and Tolerance of a New Gentamicin Collagen Fleece (Septocoll) after Surgical Treatment of a Pilonidal Sinus. Colorectal Disease, 5, 222-227. https://doi.org/10.1046/j.1463-1318.2003.00471.x

[11] Becker, M., Lorenz, S., Strand, D., Vahl, C.-F. and Gabriel, M. (2013) Covalent Grafting of the RGD-Peptide onto Polyetheretherketone Surfaces via Schiff Base Formation. The Scientific World Journal, 2013, Article ID: 616535.

https://doi.org/10.1155/2013/616535

[12] Huang, R.Y.M., Shao, P., Burns, C.M. and Feng, X. (2001) Sulfonation of Poly (Ether Ether Ketone) (PEEK): Kinetic Study and Characterization. Journal of Applied Polymer Science, 82, 2651-2660. https://doi.org/10.1002/app.2118

[13] Harting, R., Barth, M., Bührke, T., Pfefferle, R.S. and Petersen, S. (2017) Functionalization of Polyethetherketone for Application in Dentistry and Orthopedics. BioNanoMaterials, 18. https://doi.org/10.1515/bnm-2017-0003

[14] He, S., Zhai, S., Zhang, C., Xue, Y., Yang, W. and Lin, J. (2019) Effect of Sulfonation Degree and PVDF Content on the Structure and Transport Properties of SPEEK/PVDF Blend Membranes. Polymers, 11, 676. https://doi.org/10.3390/polym11040676

[15] Bishop, M.T., Karasz, F.E., Russo, P.S. and Langley, K.H. (1985) Solubility and Properties of a Poly(Aryl Ether Ketone) in Strong Acids. Macromolecules, 18, 86-93. https://doi.org/10.1021/ma00143a014 